\title{
VARICES EN LAS EMBARAZADAS $\left(^{\circ}\right.$
}

\author{
Doctor J. M. Oviedo G.
}

Líbano - Tolima.

Cuando llega al médico una señora en embarazo, a consultar para las molestias que se le presentan en sus piernas varicosas. el médico se halla ante un caso que parece de una trivialidad ciesconcertante. Cree que no tiene más que limitarse a aconsejar medias elásticas, vendajes, reposo y determinada posición para las piernas, dejando en ella la impresión de que no hay nada que hacer en esa época, debe aguardar a que pase su puerperio para darle solución. Porque el público conoce cómo deja do ser problema una vez concluído el emberazo; si sus venas continúan varicosas no presentan la sintomatología actua! y se hacen más lievaderas, por lo menos, hasta que no se presente otro embarazo. Pero hay casos, en general numerosos, donde las várices constituyen una verdadera enfermedad, por variada cantidad de sintomas y trastornos, que pueden conducir hasta la incapacidad, que no se pueden pasar por alto, dejando para otra época su solución; sino iniciar el tratamiento buscando un resultalo definitivo o para dar un alivio mientras se pueda acometer uno ra dical. Para estos casos, muy numerosos, de corriente observación, es donde el obstetra se encuentra en una posición que estımó. está por resolver. Estas líneas son un aporte mínimo a esa solución, que sin pretender dar una pauta definitiva sí pueden ser el comienzo de poder trazar una. conducta recta en la interpretación y trätamiento de dolencías tan frecuentes. Este trabajo resume las experiencias de varios autores cuyas prácticas ize seguido, modificándoles para obtẹner unidad y es, además, 12. corrección de mis fracasos y errores iniciales, se fundamenta sobre un numero reducido de casos, pero suficientes como para sacar conclusiones.

No: son muchos los trabajos que tratan de la etiopatogenia o cie la terapéutica de las várices venosas durante el embarazo.

(*) Trabajo presèntado, por el autor, al III Congreso Colombiano de Obstetricia y Ginecología, reunido en Ibagué. 1957. 
pero siendo muy alta su frecuencia, es importante para el médico práctico. Basta con recordar que las estadisticas dan un 25\% de várices para las primíparas y de un 50\% para las multiparas, aunque algunos estiman que ese porcentaje se puede elevar hasta un $75 \%$. Los tratodistas lo enfocan como un problema general, sin detenerse a considerarlo durante el embarazo, que es la época durante la cual es muy conocida su exacerbación y desarrollo. Es alto también el porcentaje de embarazadas que desde los primeros momentos notan la distensión extraordinaria en las venas de los miembros inferiores, siendo en algunas, éste uno de los primeros signos que les indica su estado de gestación. Las várices pueden ocupar todos los puntos del sistema venoso, pero las que interesan para este trabajo son las que se hallan en los miembros, inferiores, en las venas genitales y en los plexos hemorroicales. En los grandes labios, clitoris, ias ninfas y en el territorio vascular vulvo-genital, se presentan várices que pueden alcanzar las proporciones de verdaderos tumores, siendo su etiopatogenia la misma.

Historia.-Hipócrates, 460 años antes de Cristo, hacia tratamiento para las várices. que consistia en punciones, por creer que se hallaba ante un fenómeno estático, es decir, desconocia la circulación de la sangre. Aurelio Celso, 30 años después de Cristo, en su "Tratado de Medicina", trataba las várices por medio de diéresis de los tejidos. Galeno (131-200), hace la anotación de que por las venas cursaba un elemento líquido impulsado por el corazón $y$, por medio de extracciones con ganchos, les hacía tratamiento. Paulus Aegineta, en el siglo VII, recomienda la ligadura y sección de las várices y pide que la intervención se haga del lado interno del muslo, o sea el mismo problema de nuestros días. Ambrosio Paré, (1510-1590), anota que la úlcera de la pierna tiene directa relación con las várices. Andrés Vesalio (1514-1564) estudia las válvulas venosas, pero no explica su funcionamiento. William Harvey, es quien tiene el mérito de haber descrito la circulación de la sangre. Von Loder (1803) hace el estudio de la red venosa, describe las comunicantes y muestra los defectos que se presentan cuando ellos son incontinentes. Carlos Pravaz (1791-1853) ideó la jeringa que lleva su nombre con el fin de introducir sustancias dentro de los aneurismas, trajo como causa el hacerlo también en las venas varicosas. Es desde esa época que el tratamiento ha alcanzado notorios adelantos.

$\mathrm{Me}$ parece necesario recordar algunas nociones de anatomía, porque ellas son indispensables para una mejor compren- 
sión y dan claridad suficiente sobre la caprichosa distribución de la red venosa de los miembros inferiores, como también actualizan nociones fundamentales para explicar la etiopatogenia de esta afección.

Anatomía.-Las paredes de las venas son más delgadas que las de las arterias, por esta causa permiten ver por transparencia el color oscuro de la sangre, cuyo color está en proporción con él porcentaje de hemoglobina reducida. Son conductos casi rectilineos que describen curvas de gran arco.

Los tratadistas están de acuerdo en comparar el sistema venoso de la pierna como un "cono", cuya base está formada por innumerables venulas que recibe la sangre del lecho capilar y cuyo vértice está constituído por la vena femoral, que al pasar bajo la arcada crural adquiere el nombre de vena ilíaca externa:

Con un fin, más que todo didáctico, se divide esta red venosa en tres sistemas: $10^{\circ} \mathrm{El}$ superficial, formado por las venas: safena interna y externa; el profundo, formado por la vena femoral, que lo agrupan o denominan con el nombre genérico del "sistema de la vena ilíaca externa". 2 o El sistema de las ramas extrapelvianas de la vena ilíaca interna (la vena obturatriz, la glútea, la pudenda interna y la vena isquiática). $3^{\circ}$ El sistema de las venas comunicantes.

El sistema superficial.-Este sistema ofrece una anatomía variadísima que hace contraste con la relativa fijeza del sistema profundo, ésta es causa de crear dificultades para hacer un estudio detalladio. Un ataque varicoso, de cualquier intensidad, sobre cualquier grupo de venas superficiales, modifica no solo la topografía, para cada caso, en forma que hace, a veces, dificil reconocer a cuál sistema corresponde. La misma visibilidad está sujeta a varios factores, como sería la transparencia de la piel y se ven casos de personas con poco pigmento de la piel, que solicitan tratamiento para una red venosa muy visible sin que tengan várices verdaderas.

El sistema profundo o de la vena femoral.--Está formado por las colaterales dorsales de los dedos, la interoseas dorsales, la vena pedia, que forma la tibial anterior, la vena tibial posterior, las peroneras anterior y posterior que forman la peronera, la poplitea que recibe la safena externa y que se transforma en vena femoral al pasar el anillo del tercer aductor. La vena femorai recibe dos colaterales de importancia: la vena femoral profunda 
por su cara posterior a unos 4 a 6 centímetros de la arcada crural y la vena safena interna por su cara anterointerna, y a 3 o 4 centímetros de la misma arcada.

El sistema profundo corre por entre espacios intermusculares - subaponeuróticos que le ofrecen apoyo externo. Las venas superficiales están colocadas dentro del tejido celular subcutáneo. dentro de la grasa y cubiertas por la piel, en esta forma, sin ningún apoyo, no pueden ofrecer más resistencia que la de la pared venosa para impedir las dilataciones. Esta es una de las primeras razones para explicar la frecuencia de las várices en el sistema de las venas superficiales.

Se tiene que convenir que las descripciones que traen los libros son puramente esquemáticas y hasta cierto punto convencionales, porque son tantas y tan variadas las irregularidades del sistema superficial que se hace un esfuerzo inútil querer acomodarlo a un solo patrón, por ésto no hay que tener como fijas un número determinado de colaterales, como nunca se debe de olvidar las caprichosas combinaciones. En el caso de la safena interna, por ejemplo, se hacen descripciones anatómicas dándole un curso más complicado y caprichoso del que comúnmente se le atribuye. Se describen dos safenas internas: una superficial y otra profunda; pero en el vasto territorio avenado por la safena interna, es donde se asientan la mayoría de las váricess del miembro inferior.

El sistema de las ramas extrapelvianas de la vena iliaca interna o hipogástrica, tiene también una caprichosa anatomía, y el olvido de su existencia es causa de fracasos al intervenir sobre la vena safena interna.

El sistema de las venas comunicantes.-El sistema superficial y el sistema profundo se hallan comunicados entre sí por medio de venas comunicantes y de venas perforantes. Distingue a las primeras, el ser conductos que unen directamente una vena superficial con otra profunda y donde el sentido de la corriente es de la superficie hacia la profundidad. Las venas perforantes tienen otra disposición, atraviezan la aponeurosis profunda de la pierna, sin buscar los planos de clivaje intermusculares, son por lo tanto, puras venas que nacen en los cuerpos musculares por debajo de la aponeurosis se dirigen a desembocar en el sistema superficial y no constituyen una comunicante directa de ambos sistemas. Su importancia en la patogenia de las várices es nula, mientras que la de las comunicantes se hace a veces pri- 
mordial. La mayoría de las comunicantes se dirigen de una de las safenas o de sus ramas hacia las venas profundas (tibial anterior y posterior, peronera), son relativamente numerosas (la safena interna y la femoral tienen 2 a 3 ); hay que tener presente que muchas venas comunicantes, sobre todo en las piernas, rio abocan directamente en las venas superficiales sino en otras secundarias alejadas de aquéllas; en consecuencia, la extirpación de las venas safenas, en forma completa por sección o fleboextracción, no puede poner a cubierto de las recidivas, pudienco quedar un lago venoso formado por venas superficiales secundarias que se llena por una comunicante incontinente.

El sistema venoso está dotado de válvulas (1) colocadas de trecho en trecho (2), conjuntivo-endoteliales con una disposición tal que pueden ser franqueadas cuando la corriente se dirige hacia la raiz del miembro y que la impiden cuando se invierte el sentido de su curso. Su número es muy variable, hallándose más en las venas pequeñas que en las grandes y en las venas profundas que en las superficiales. Los anatomistas están de acuerdo $\epsilon \mathrm{n}$ sostener que las venas cava inferior y la ilíaca primitiva no. poseen válvulas y que el peso de la columna. líquida recae sobre las de las venas iliaca externa y las de la parte alta de la femoral; pero se debe dejar constancia de que hay un cierto número de casos donde estas válvulas faltan, dando una de las causas o de las posibilidades para el desarrollo de las várices en el miembro inferior.

Anatomía patológica.-Ya es sabido que las paredes venosas están constituídas por tres capas cuyas modificaciones se van estableciendo a medida que el proceso varicoso aumenta. La más atacada es la intima, que aumenta de grosor, que en algunos casos es uniforme en sentido circular, en otros lo hace por segmentos. Es debido al aumento del tejido conectivo y está relacionado directamente con la presión aumentada y constante a que se encuentra sometida la pared venosa. Esta capa, lo mismo que la porción más interna de la túnica media, se nutren por imbibición; si ella se altera se acumulan metabolitos que aumentan la permeabilidad capilar, pasando a la intima plasma sanguínea que se traduce en edema de la misma; hày una intensa proliferación conectiva que hace desaparecer los elementos celulares, pudiendo llegar a la calcificación. Esta esclerosis produce en el endotelio sinuosidades en la pared, de donde no es infrecuente el desprendimiento de trombos. En este estado de la intima, se engrosa y endurece y colabora progresivamente en la pro- 
ducción de várices, haciendo rígida la pared y retrayendo las válvulas ya incompetentes por medio de la estasis venosa. La túnica media presenta anoxemia que la vuelve fibrosa, sus fibras musculares se disocian, el tejido elástico se condensa en algunos sitios y viene a ser reemplazado por tejido conectivo en hiperplasia. La adventicia es afectada por este proceso de proliferación conectiva, pero la causa radica más en procesos inflamatorios de poca actividad, que no son otra cosa que la expresión de los comunes procesos inflamatorios dérmicos, tan comunes en Ios varicosos; esta periflebitis tan común es la que explica las fuertes adherencias que siempre hay entre los tejidos vecinos y los cordones varicosos.

El término "várice" se limita en su acepción ordinaria a las dilataciones localizadas que se escalonan a lo largo de ciertas venas, pero como no siempre coincide la enfermedad con esta concepción macroscópica, también se engloban en ella y se acepta con la categoría de venas varicosas las flebectasias o dilataciones cilíndricas uniformes. Este unicismo existe en la patogenia, no así en la morfología, que tiene como única causa la presión intravenosa aumentada con incompetencia valvular que conduce a ambos fenómenos. Morfológicamente las venas varicosas aparecen como unos cordones rígidos uniformemente dilatados o como cordones de desigual calibre y resistencia; hay por lo tanto, áreas de paredes de delgadez extrema y otras zonas conde prima la dureza y rigidez, dando el aspecto de rosario.

Esta forma morbosa aún no ha recibido una definición en la cual se hallen totalmente los diferentes tratadistas de acuerdo. Hay la tendencia de aceptar la definición antigua que hace distinción entre las flebectasias transitorias y várices verdaderas con alteración permanente de la pared venosa; pero se puede aceptar "que ellas son la dilatación segmentaria patológica de los vasos venosos de alguna región, que no es exclusiva del embarazo, durante el cual, sin embargo, es muy frecuente".

Fisiologia.-En el sujeto normal la circulación venosa en las piernas es centripeta. De los capilares la sangre se dirige por los tres sistemas descritos hacia la raiz del miembro para alcanzar la. vena ilíaca y la vena cava inferior y luego la auricula derecha. Se debe dejar constancia que es por el sistema profundo por donde se moviliza un mayor volumen de sangre.

Múltiples son los factores que intervienen para imponer una progresión centrípeta de la corriente venosa; los podemos enu- 
merar así: 19 La presión negativa intratorácica, que atrae el contenido de la vena cava inferior durante la inspiración; ese efecto aspirativo tiene una mayor importancia en las venas que afluyen a la cava superior y se ve contrarrestado por el aumento de la presión intra-abdominal que acompaña el descenso del diafragma. $2 \%$ La presión intermitente ejercida por las contracciones de los músculos estriados del miembro sobre las venas y en particular de las profundas. Los músculos de la locomoción forman una especie de estuche a los vasos venosos. de tal manera que al entrar en funcionamiento exprimen el contenido de éstas hacia la raiz del miembro; cuando entran en reposo, las venas profundas se encuentran semivacias y con una presión venosa disminuída, que trae una aceleración de la corriente periférica $y$ del sistema superficial por intermedio de las venas comunicantes. Es, por lo tanto, el sistema profundo el único que se beneficia con las contracciones musculares; el superficial no tiene este factor porque la piel o el tejido celular subcutáneo no puede prestarle ningún apoyo, propiciando la facilidad de su dilatación y la deformación de las válvulas. Esto explicaría el por qué se encuentran más varicosos en individuos que tienen que permanecer de pies, como los peluqueros o los odontólogos, que en los que su profesión los obliga a caminar: los carteros o los mensajeros. 3 o La capacidad contráctil de la pared venosa, en particular en las vénulas, es otro factor de progreso de la sangre en las extremidades inferiores. 40 $\mathrm{E} 1$ "vis a tergo" (lat. vis.: fuerza tergun: atrás), que es la fuerza sistólica ventricular que resta después de haber atravesado los vasos capilares, no es mucha su importancia. 50 La acción de las válvulas que impide la acción de la gravedad y se opone a que la sangre regrese y asi se pueda perder el terreno ganado.

Fisiopatología.-Cualquiera de los sistemas pueden ser afectados por las dilataciones venosas o por insuficiencias valvulares, pero ellas se encuentran de preferencia en las comunicantes o en el sistema superficial. Una primera fase es de dilataciones o de estancamientos sanguíneos, es decir, disminúción del desagüe ascendente normal. Este fenómeno es de difícil interpretación en la actualidad, pero los tratadistas se inclinan a explicarlo por la pérdida del tono de la pared venosa y su consiguiente dilatación primaria son debidas probablemente a una debilidad mesodérmica o a trastornos endocrinos mal comprendidos hasta el momento. Un poco más adelante la distensión venosa provocará la separación de los bordes valvulares y las hace in- 
continentes (B: positivo). Existe una tercera variedad y es aquella donde las válvulas de la safena están intactas, pero una o varias colaterales son incontinentes; se produce en este caso un flujo sanguineo de dentro hacia afuera (blow-out) (B. negativo). También se puede presentar que ambos mecanismos estén en juego, que sean incontinentes las válvulas de la safena y de una a varias comunicantes (B. doble).

Antes de pasar adelante me parece indispensable dar a conocer cómo se establece, por medio del examen de la paciente, cuál de las tres diferencias de lesión venosa se halla en juego. Para ello se recurre a la prueba de Brodie-Trendelemburg. En un primer estado esta prueba será igual a cero (0); únicamente se trata de la pérdida del tono de la pared venosa y su consiguiente dilatación primaria; la colocación de un lazo proximal no altera el estado de dilatación venosa o sea la incontinencia valvular, el signo Brodie-Trendelemburg será positivo. La colocación de un lazo proximal después de haber vaciado las venas por levantamiento del miembro, al ponerse de pies la paciente y mantenida la presión, las venas permanecen vacías, pero se llenan bruscamente cuando se retira el lazo. La 3ạ variedad está caracterizada porque las válvulas de una o varias colaterales son incontinentes. En este caso, poniendo la presión del lazo en el tercio superior del muslo y al ponerse la paciente de pies, las venas se llenan velozmente: primero en el terreno de la comunicante incontinente y al retirar la ligadura no se nota aumento en el tamaño o de tensión de las várices por falta de flujo vertical descendente de la safena interna. El signo de Brodie-Trendelemburg es negativo. Por último, se puede presentar que las válvulas de la safena y las de las comunicantes sean a su vez incontinentes; en este caso, una vez vaciadas las venas y colocado el lazo, al ponerse de pies la paciente, las venas se llenan rápidamente, pero quitado el torniquete se nota un mayor relleno, ha funcionado la columna sanguínea de la safena interna. El signo de Brodie-Trendelemburg es en este caso doble.

Pero cualquiera que sea la modalidad del signo de BrodieTrendelemburg, la sangre no hace un intercambio correcto de oxígeno en los tejidos y por la rémora venosa permanente, ella está cargada de anhídrido carbónico; esta falta de oxígeno explicaría la cianosis y disminución de la temperatura local que es tan apreciable en todos los varicosos y las muestras de nutrición insuficientes, notable en la piel y en las faneras; ello es más notorio con un signo de Brodie doble; las corrientes sanguíneas 
se encuentran, hay un reflujo venoso horizontal y vertical, una infiltración o trasudación de suero, una imbibición de los tejidos, una pérdida de la vitalidad de los mismos, y naturalmente si hay un traumatismo o se presenta una infección, entonces en este terreno abonado se presentará un campo propicio para el desarrollo de una úlcera varicosa.

La presión venosa es variable: ella depende de factores generales o regionales. La insuficiencia cardiaca y las pericarditis aguda's o crónicas serian el producto de las primeras, una compresión extrínseca a causa de una tromboflebitis o flebotrombosis obrarian como causa local. Varios autores han determinado que la presión venosa en cualquier punto del miembro inferior está demostrada por la distancia que media entre ese punto y la auricula derecha, es decir, que depende de la acción de la gravedad manifestada por la longitud de la columna líquida existente entre los dos puntos. Es muy poco apreciable la diferencia de presión, estando de pies, entre un varicoso y otro sano, pero la presión venosa en decúbito dorsal de un varicoso, tanto en las várices como en la vena cubital, es sensiblemente mayor que la de un sujeto testigo no varicoso; se explica este fenómeno por el aumento del volumen sanguíneo que produce la entrada en circulación de la sangre retenida en las várices; se debe pensar que un tratamiento dirigido a curar las várices y eliminar los grandes lagos venosos, por un efecto secundario mejoraría el estado funcional cardíaco de un enfermo.

La presión de la vena safena interna se ve modificada y presenta cambios cuando varía la presión intra-abdominal; se puede demostrar fácilmente cuando se hace "pujar" a la paciente, porque hay un reflujo centrífugo en la vena safena interna $y$ en la cava inferior con incompetencia valvular. Por medio del signo de Adams, que es.simplemente hidrostático, se demuestra: palpando el cayado de la safena interna con la enferma de pies, se descubre un thrill o frémito-venoso, cuando la enferma tose - puja; hay aquí una válvula suficiente, da el signo de Brodie negativo para detener la acción de la gravedad, pero se contiene ante la acción combinada de la gravedad y de la presión intra-abdominal.

La posición del miembro determina el volumen de la pierna de las varicosas. At pasar de la horizontal a la posición de sentada con la pierna colgando verticalmente, después de una hora, se nota el aumento; pero permaneciendo de pies y estacionaria el aumento es mucho mayor ( $1.1 \%$ primer caso, $3,6 \%$ segundo); en 
cambio si de esa postura se pasa a caminar por una hora, el volumen disminuiría nuevamente $(1,9 \%)$.

Muchos tratadistas descartan aún la posibilidad de que puedan existir alteraciones de los capilares arteriales, y otros, en cambio. lo afirman y hasta han establecido grados de diferenciación. Creo que indudablemente deben existir esas lesiones; bastaría con pensar que cuando se ha establecido una corriente sanguinea retrógrada que vence el obstáculo arteriolar por medio de la hipertensión venosa, esos capilares deben de participar de las alteraciones y estar modificados.

Etiología.-La causa de las várices es múltiple. Es la acción sostenida de distintos factores cuya intervención varia de importancia de uno a otro caso.

En primer término se debe hacer mención de la predisposición hereditaria, cuya influencia resalta como dato en la amnannesis de cada caso; uno de los padres aparece con antecedente varicoso. La debilidad heredada se localiza en los tejidos de origen mesodérmico, explicando asi la existencia en los varicosos de hernias, hemorroides, varicoceles, pies planos, hábito asténico e hipotensión arterial.

Los "trastornos endocrinos" influyen en el estado de tonicidad de la pared venosa. Es el caso de pensar que la tendencia familiar anotada antes tiene como base la coexistencia de disendocrinias en varios miembros de la familia; a este factor hay que darle una mayor importancia; los tratadistas esperan hallar en ella la respuesta a la solución causal de esta incógnita terapéutica. Basta tener presente las alteraciones del equilibrio endocrino respecto al tono venoso; cuando se ve que en los primeros meses de embarazo ya comienzan a notarse várices en las piernas, no pudiéndose alegar una compresión ejercida por el útero sobre las venas abdominales, cava e ilíacas, ni seria justificable achacarlo a un aumento de la presión intra-abdominal de aparición precoz. Pero hay una razón más que no se puede pasar por alto: es la sostenida por las enfermas, que su proceso varicoso apareció durante los cambios de estado hormonal: el embarazo, la menopausia y en ocasiones en la pubertad. Hoy se acepta la existencia de un factor constitucional que determina una particular tonicidad para los tejidos en cada organismo, que engendraría la debilidad de la pared venosa para dilatarse hacia afuera 0 por un defecto de sostén de los tejidos que la rodean. En el embarazo hay exageracion de la presión endovenosa en los miem- 
bros inferiores por aumento del volumen circulante (Hidremia); además no se puede olvidar el reblandecimiento general ce todos los tejidos orgánicos para darse cabal cuenta de que durante el periodo gestatorio hay reunión de las condiciones optimas para que las venas sufran dilataciones varicosas.

La posición "erecta" que hace recaer una presión muy superior sobre las paredes venosas del miembro inferior, que la soportada por las demás venas del organismo, con excepción del campo vascular hemorroidal, que aclara por qué son más afectadas con estas novedades. De aqui ha nacido una teoria que se puede llamar anatómica, para explicar la formación de las várices: al estar desprovistas de válvulas las venas cava inferior e ilíaca primitiva, el peso de la columna sanguínea recae sobre las válvulas de la ilíaca externa, que en muchos casos tambien hacen falta, soportando la integridad de ese peso las válvulas de la femoral, que en ocasiones por encima del cayado de la safena. interna se halla desprovista a su vez de ellas; esa sobrecarga se transmite directamente al árbol venoso superficial, aumentando la posibilidad de la aparición de várices. Las autopsias han demostrado la desaparición de válvulas en un miembro más que en el otro, que sería una explicación para la unilateralidad de afección.

El "aumento de la presión intra-abdominal", que puede deberse a causas orgánicas, como en los últimos meses del embarazo, a la existencia de tumores abdominales y pelvianos que procucirian várices por compresión, otras causas son atribuíbles a la tos, a los pujos, a la constipación y a la disuria. La hipertensión venosa durante el embarazo, que aumenta la presión intraabdominal y la correspondiente congestión pélvica característica de este estado. Las modificaciones de la posición del corazón durante el embarazo para la aspiración venosa: la disminución de la actividad muscular de la mujer embarazada. El factor hereditario, que no sería dominante en el sentido mendeliano, sino recesivo con predominancia en el sexo femenino, las estadísti-. cas establecen un $62,3 \%$ de embarazadas con antecedentes familiares positivos para las várices.

Las "infecciones agudas o crónicas de la pared venosa", que $\in n$ ocasiones son determinantes en la producción de várices. $\mathrm{Su}$ acción puede ser en forma indirecta, como en una flebitis de las venas profundas que obra repercutiendo sobre el sistema superficial donde se producen dilataciones como fenómeno compensatorio. 
Las "fístulas arteriovenosas congénitas", que pueden provocar el desarrollo precoz y rápido de várices, originadas por la persistencia o por defecto en el cierre de las comunicantes vasculares del periodo fetal, que generalmente se ocluyen antes del nacimiento, son las falsamente llamadas "várices arteriales". En ccasiones se cierran por una simple barrera celular o imperfectamente que se reabre por un simple esfuerzo muscular o en la época de la pubertad.

Sintomatologia.-El dolor es el síntoma que más llama la a.tención de las enfermas, el que les hace llegar a consulta. Es de intensidad variable, molesta más a la enferma por su persistencia que por su intensidad. Cuando se hace intolerable hay que pensar en una flebitis, en una erisipela o que se vaya a abrir una úlcera de la pierna. Su descripción es muy variable: unas lo sienten como un calambre; para otras es algo sordo y profundo, localizado en los músculos de la pantorrilla; algunas. io sienten más de noche que de día, lo localizan generalmente en la pierna, pero para algunas lo sienten en todo el miembro inferior y hacen llegar hasta los dedos. Otro tipo de dolor común a las várices es el que sienten sobre el trayecto de las venas más dilatadas y más cercanas a la piel: son punzantes o simplemente se trata de una mayor sensibilidad al golpearse o al simple rozamiento de los vestidos. También sienten acortamiento o estiramiento de las venas $\mathrm{y}$ en ocasiones hay dolores verdaderamente terebrantes.

El dolor se hace presente desde la iniciación de las várices; tiene periodos de intensidad o de disminución, hay un aumento €n los días premenstruales, pero disminuye durante él; también aumenta durante el embarazo. En cada enferma existe un tipo especial de dolor: en unas lo sienten por las mañanas y cesa al entrar en actividad, pero una inmensa mayoría lo sienten más por las tardes cuando dejan de estar activas y él será tanto más intenso cuanto la actividad haya sido desarrollada de pies.

. Las que tienen várices de una sola pierna sienten la "pesadez y cansancio" del miembro varicoso más nitidamente al hacer contraste con su pierna sana.

La aparición de "parestesias" en las várices se traducen por una sensación de hormigueo de la pierna o del pie; en otras ocasiones hay ardor o sensación urente sobre la vena afectada o sienten una especie de corriente líquida caliente que les hace pensar no pocas veces que su várice ha estallado, produciendo una hemorragia externa. 
No hay ninguna relación entre las sensaciones dolorosas o su intensidad y las várices existentes. En enfermas portadoras de várices pequeñas y profundas, tienen a veces molestias que les causan verdaderos sufrimientos: mientras que otras con voluminosas várices y enormes lagos se quejan únicamente de su aspecto antiestético.

El "edema", demostrable fácilmente en un gran número de varicosas, comienza en la generalidad de los casos sobre el dorso del pie y en los tobillos, borrando los relieves maleolares; es consecuencia directa del aumento de la presión hidrostática. Es blando y plástico a la presión. Se presenta con desarrollo desigual en ambas piernas, conduce a alteraciones tróficas de la piel.

La "cianosis" es causada por la permanencia de la sangre por más tiempo del normal, por pérdida cada vez mayor de oxígeno y aumento de hemoglobina reducida.

Los "trastornos tróficos de la piel" son causados también por la rémora venosa y a la compresión de las arteriolas y vénulas por el edema. Son tejidos anóxicos y por consiguiente mal nutridos que llegan a las alteraciones de la piel, del tejido celular subcutáneo, eczema varicoso, esclerosis, atrofias y cicatrices. pigmentaciones, úlceras varicosas, las periostitis como consecuencia de ellas, flebitis y periflebitis, las dermoepidermitis y hasta las hemorragias subcutáneas y externas.

\section{SEGUNDA PARTE}

Las várices se encuentran entre las afecciones que deben considerarse como de las más frecuentes. Parece que un 20\% de la población sufre de ellas; siendo la población femenina la: que da un porcentaje más elevado; no es de extrañar si se piensá que el embarazo es un factor etiológico por su doble acción. hormonal y mecánica.

Una gran mayoría de las enfermas llegan a la consulta para solicitar una opinión médica para sus estados varicosos por razones de estética o por la modalidad de los vestidos. Esto es una confirmación del criterio simplista imperante sobre la poca atención que se ha dado entre nosotros a estos estados varicosos respecto a su tratamiento.

La edad de las consultantes es factor importante: la mayoria lo hacen entre los 20 y 60 años, pero para las que se relacionan con el embarazo las edades se hallan comprendidas entre los 18 y. los 42 años; entre ellas hay muchas que las presentan desde 
la primera gestación, desde los primeros meses presentan dilataciones venosas en sus miembros inferiores.

Es un error creer que las várices son una enfermedad de la vejez. Un alto número de personas las comienzan a padecer desde la adolescencia y juventud. Tampoco pueden considerarse como dependiente exclusivamente del embarazo, porque las presentan mujeres que no han tenido hijos, pero la influencia de la gestación no puede ponerse en duda.

La consulta para várices se hace generalmente en forma tardía, cuando las han soportado por varios embarazos y ya las afecciones dermatológicas están muy adelantadas, con lesiones circulatorias o tróficas casi irreversibles, haciendo muy difícil el tratamiento.

Hay un gran número de enfermas para quienes transcurre la vida sin que se hagan ningún tratamiento. En la generalidad no quieren someterse a curación sino para un solo miembro o sea el que les causa molestias, pero en la casi mayoría de los casos se descubre por el examen la bilateralidad de la afección.

La red venosa superficial es particular para cada paciente, acentuándose en las pacientes con várices, su dibujo es muy variable y se puede afirmar que no hay tipos especiales en donde se puedan agrupar, es cambiante hasta con la posición que se le dé al miembro y aun asi hay que aguardar un tiempo prudencial para obtener un relleno visible y estable. El examen detenido de cada varicosa es indispensable, cuanto más tiempo se le dedique a su estudio da mejores resultados. Cada caso es independiente de otro y tiene una fisiopatologia individual; por eso no puede haber tipo de tratamiento estandard o preconcebido. porque nos llevaría al fracaso; hay, por lo tanto, que fundarlo sobre un estudio clínico completo. Cada varicosa presenta dificultades múltiples y de su exacta interpretación sale un buen tratamiento. Pero hay muchos casos en que la clínica sola no es suficiente, la dificultad está representada por el estado del sistema venoso profundo, cuando hay secuelas de flebitis anteriores o por falta de venas superficiales fácilmente visibles por un panículo adiposo abundante. Para estos casos la flebografía es indispensable. Sorprende a veces ver una gran red varicosa profunda en enfermas que únicamente presentan pequeños paquetes visibles, ante la cantidad que se obtiene por medio de la flebografía.

El diagnóstico de las várices es sịn duda de una desconcertante simplicidad. Es la enferma que nota el cambio del volumen 
de sus venas, pero ignora que los trastornos que sufre tienen su causa en esa dilatación. El médico que examina el caso tiene el diagnóstico a la vista. Es el más fácil que nos ofrece la clínica; éste es por lo menos el concepto general; pero algo más difícil se hace en las enfermas cuyas várices están ocultas por un panículo adiposo abundante, por el edema tan común a las varicosas y durante el embarazo, por dermatoesclerosis o por una elefantiasis.

Durante el embarazo hay que tener presente la poca relación que pueda existir entre la magnitud del cuadro varicoso y la sintomatología que éste pueda tener realmente, es con la continua observación y la experiencia obtenida como se puede hacer esta diferencia. Durance el embarazo las enfermas se quejan de dolores en las piernas, aun no siendo varicosas. No.se puede olvidar que várices y pie plano son de frecuencia conocida, con sintomatología similar, que hace indispensable distinguir lo que corresponde a cada entidad y hacer el tratamiento para cada caso.

Se debe recordar que hay un gran número de enfermas que tenian várices antes del embarazo, es decir, eran preexistentes, cuando ellas parecen en edad temprana, que sufrirán posteriormente la acción de las gestaciones y sus repercusiones sobre sus venas; en estos casos se establece una Iucha entre el médico y la afección, hay que aplicar los recursos terapéuticos más aconsejables y evitarle a la paciente largos periodos sin control.

Las várices son una afección de tipo progresivo; por єsto no se puede asegurar que una enferma correctamente tratada no vuelva nunca a tener várices; en ella hay un terreno predispuesto, persiste la debilidad mesenquimática, que aun eliminando el factor mecánico-funcional como etiológico; otras venas que eran normales anteriormente pueden volverse varicosas por sufrir el mismo proceso evolutivo.

Es indispensable una buena constancia de la paciente y del médico para instalar los tratamientos oportunamente. De esto, se saca la conclusión que no se puede hablar de recidivas, si únicamente se trata de venas que eran normales y que al no ser tratadas con el tiempo lo hacen.

Tratamiento.-Existen medidas preventivas y curativas. 'Las primeras son aconsejables en enfermas que comienzan a notar la aparición građual de dilataciones venosas en sus piernas o que sufren de alguno de los síntomas descritos. Son beneficiosas por cuanto retardan la evolución progresiva de la afección. 
Se prohibe el uso de ligas, que impide la progresión de.la sangre de las venas superficiales. Se aconseja el reposo en decúbito dorsal varias veces al dia con las piernas a nivel superior a. 1 the la cabeza; así se rompe la acción del reflujo venoso centrifu६o. Las vendas y medias elásticas. Los medicamentos con productos a base de extractos glandulares que actúan sobre la tonicidad de las fibras musculares lisas. La eliminación de factores tales como la tos crónica, la constipación, la disuria, los tumores abdominales y particularmente los colocados en la esfera genital.

El tratamiento médico de las várices es paliativo cuando se presentan estas condiciones: cuando las várices son de carácter temporario, cuando la afección no ha alcanzado àu un grado ce desarrollo que permita tratamiento activo, cuando se halle contraindicado temporal o definitivamente por causas generales o locales y cuando la enferma se niega a dejarse tratar.

Los medios usados son: el reposo, que favorece la absorción. de los edemas, la turgecencia venosa y calma el dolor producido por las neuritis y mejora la circulación en las úlceras crónicas. Fl masnje local (masoterapia), que tiene efecto sedante y es eficaz parา. los :alambres y aumenta la tonicidad de las paredes venosas, facilita. los intercambios tisulares y la reabsorción de los edemas. El vendaje: existen varios tipcs de tejidos destinados a la contención elástica exter:a de la pierna elaborados en forma de medias o vendajes. Las vendas son más ventajosas por adaptarse a cada caso particular y su aplicación no demanda tiempo. Cuando hay úlceia se pueden incluir dentro de sus pliegues esponjas $u$ otros implementos que facilitan el tratamiento y curación (Met. de Nobl.).

Los medicamentos recomendados son: el embarazo trae un desarrollo progresivo de las várices que se puede modificer por medio de la administración de vitamina $C$., que tiene acción sorre las paredes vasculares. La vitamina P. (rutina o hesperidina), cuya acción sobre la fragilidad y permeabilidad capilar influye favorablemente sobre las pigmentaciones y petequias. La hanmamelis, el castaño de Indias y la nuez vómica: parece que mejoran más subjetiva que objetivamente, se indican como constrictores vasculares periféricos. Es indudable que los productos que tengan extractos glandulares unidos a vasoconstrictores serán los más apropiados; su indicación está basada en la innegable pariicipación que tienen las hormonas sexuales sobre el estado de las venas en las enfermas con tendencia a formación de várices. 
La aplicación de sustancias estrogénicas en las embarazadas da resultados sorprendentes, obraria controlando el alto nivel de progesterona circulante, al relajar la musculatura lisa, favorece la dilatación de los ureteres, vesicula biliar, conducto digestivo y también las venas. En estos últimos tiempos se ha visto que la combinación de estrógenos y progesterona produce mejores resultados. Este tratamiento se establece cuando en los últimos meses de embarazo las molestias se hacen tan notorias que la enferma se hace una inválida. Las dosis de estrógenos deben ser bajas, no son aconsejables dosis altas; generalmente se emplea el benzoato de estradiol por vía parenteral, 1-2 miligr zmos por una semana y después 1 miligramo cade tercer día por varias semanas. Estas dosis no provocan abortos o partos prematurus, ni tienen otros efectos secundarios. La hormona tiroidea en pequeñas dosis puede ejercer una acción ccadyuvante.

El embarazo no constituye contraindicación para el trat.j. miento médico de las várices, sin excluir en muchas ocasiones, de acuerdo con la sintomatología, el quirúrgico. El tratamiento esclerosante es el indicado en la casi totalidad de los casos y sus resultados son siempre satisfactorios. Como norma genera! se debe iniciar en todas las enfermas cuyos padecimientos son muy acenturdos, aplicarlo antes de los seis meses de embarazo, cuando se espera que la sintomatología se agravará con el tiempo, pudiéndose ahorrar a la paciente varios meses de sufrimiento $e$ incapacidad. Pasado éste lapso, por el poco tiempo que resta de gestación, no parece aconsejable, se aplicarán otras medidas y aplazándolo para el término del puerperio.

El médico dispone de dos métodos para la terapéutica de las várices: el esclerosante simple y el quirúrgico (esclerosante combinado). La elección de uno cualquiera de ellos se debe fundamentar sobre indicaciones particulares precisas y proporcionan excelentes resultados con tal que se haga con criterio exacto la selección de las enfermas y se use una técnica correcta.

Me he guiado para hacer la diferenciación de cuál de los dos tratamientos se debe aplicar exclusivamente en la prueba de Brodie-Trendelemburg. Cuando ella es igual a 0 (cero), o sea que existe únicamente la pérdida de la tonelidad de la pared venosa y su consiguiente dilatacićn primaria, he aplicado el método esclerosante. Si la prueba es positiva se aplicará el quirúrgico-esclerosante, porque ya hay una incontinencia valvular. Por tanto, la conductà "térapéutica tienè que estar condicionada a los resultados bien conocidos y fijos de la prueba y no en signos in- 
seguros como el volumen de las ectasias, su número o su extensión. No hay un acuerdo definitivo sobre el método quirúrgico: para unos es el más aconsejable, pero otros lo prescriben por el gran número de recidivas y fracasos. Me parece que ambos conceptos tienen fundamentos valederos, porque hoy no existe un método que permita conseguir siempre un resultado definitivo, más si se piensa que la "enfermedad varicosa" es de tendencia evolutiva y que en los casos bilaterales existe una diátesis varicosa tan considerable que el tratamiento no puede dar ninguna garantía. Pero hay una base fundamental: es que después de un tratamiento combinado, bien practicado el porcentaje de las recidivas, se ha reducido sorprendentemente en relación con las estadísticas anteriores. Se debe dejar constancia, además, que las recidivas son de ordinario leves y con un pequeño control es suficiente para que no les cause molestias posteriores.

No se intenta tratamiento para las várices porque se estima que ellas son su complicación natural. Parece que hay un criterio que creo que al proponerlo se correría el riesgo de interferir su curso normal. Esta actitud pasiva ha producido molestias y hasta secuelas irreparables. Está demostrada la existencia de flebitis latente en muchos casos de venas varicosas, si no es tratada, se recrudece con el embarazo, por congestión venosa, trauma o estancamiento, la infección puede quedar incubada hasta el período puerperal, cuando puede aparecer como una flebitis profunda fulminante.

La escleroterapia es el método de elección que da resultados excelentes en la gran mayoría de los casos e incluso ha habido aplicaciones en los últimos meses de embarazo con pleno éxito. Aplicado con criterio exacto, haciendo una rigurosa-selección de las enfermas y practicado con técnica, da siempre buenos resultados. Para su aplicación se deben tener en cuenta estas claras condiciones: en enfermas que presenten ectasias venosas iniciales de poco volumen, limitadas a un pequeño territorio a menudo a nivel de una vena comunicante insuficiente, en casos de recidivas iniciales después de intervención quirúrgica, en algunos casos, ya sometidos a tratamiento combinado para completar la acción de la inyección esclerosante intraoperatoria y en los casos en que hay que atenuar los síntomas graves y molestos, debido a complicaciones en los que exista contraindicación para la intervención. Creo necesario que se haga una revisión sobre algunos puntos del tratamiento de las várices en las embarazadas, que piden nuevos estudios y muchos requieren ser revisados y funda- 
mentados sobre respaldo experimental; como es de esperarse, se hagan las diferencias indispensables y se pongan limites precisos entre los varios estados evolutivos, única manera de poder trazar una norma clara sobre el tratamiento para cada caso. Estas ideas son las que han creado este trabajo, por el número reducido de casos, comprende únicamente 82 , sacados de la clientela privada, no da margen para sacar conclusiones, pero si autorizan para demostrar que el tratamiento es factible y que su iniciación debe ser precoz.

La esclerosis como tratamiento no puede someterse a un patrón o tipo especial. No admite sistematización. Se debe comenzar en las ectasias más evidentes en comunicación con nódulos cercanos pequeños. No se debe hacer en las ectasias más superficiales para no determinar enrojecimientos y reacciones dolorosas, ni tampoco empezar por las cercanas a la cresta tibial o en las regiones perimaleolares, en ellas las reacciones son particularmente molestas, pudiéndose comprometer el éxito en enfermas sensibles. Se deben emplear siempre pequeñas dosis de líquido esclerosante $\mathrm{y}$ en concentraciones bajas, especialmente al comienzo y nunca llegar a dosis elevadas ni siquiera en el curso ulterior. Si la inyección no se muestra eficaz, es preferible cambiar de droga en vez de usar dosis altas o concentradas.

La elección de sustancia esclerosante tiene una importancia muy relativa. Los buenos resultados dependen exclusivamente de una técnica correcta. El intervalo de las inyecciones tampoco tiene ni puede someterse a regla fija, depende la reacción local y la distancia entre las ectasias varicosas por tratar. Se debe inyectar a intervalos largos, no son escasas las reacciones tardías, por lo tanto, antes de una nueva inyección en una ectasia ya tratada, sin resultado, hay que aguardar por lo menos 15 días. No son recomendables ni el reposo ni los vendajes, después de la inyección únicamente se coloca encima un tapón đe algodón sujeto con un esparadrapo. Nunca he aplicado más de tres centimetros cúbicos de solución por inyección, ni tampoco una concentración mayor del 5\%. Aplicando una mayor cantidad o en mayor concentración el líquido inyectado puede producir daños en las válvulas de las venas comunicantes y en la circulación profunda.

Practico la inyección con la enferma acostada. Excluyo la posición de pies o sentada para evitar lipotimias y por permitir una mejor quietud y comodidad. Es la:posición ideal por mante- 
ner las venas con poco contenido sanguíneo y contribuye a que la solución actúe sin disminuir su concentración y se mantenga en el lugar inyectado.

He usado la siguiente técnica: enferma en decúbito dorsal o ventral, según el caso. Colocar torniquete si la vena no es visible. En contados casos se debe considerar si la vena tiene demasiada sangre, entonces se levanta el miembro y una vez la distensión desaparecida de la várice, se coloca sobre la cama y se practica la inyección, Se inserta la aguja paralela al eje mayor y si se van a inyectar varias en la misma sesión se comenzará por la más lejana. Se deja entrar sangre a la jeringa que da la seguridad de estar dentro de la luz venosa. Se aisla un trayecto de la vena colocando los dedos medio y anular izquierdos haciendo presión por encima de la aguja a unos 2 o 3 centímetros y el pulgar unos centímetros por debajo, sobre la misma vena $y$ se inyecta una cantidad adecuada a la importancia de la várice. El dolor no es siempre signo de extravasación, basta con aspirar sangre para saber si es la irritación de la intima por la esclerosis. La presión de los dedos debe sostenerse por unos tres minutos. No me parece aconsejable no hacer tratamiento sino para una sola pierna, en ambas la enferma no puede caminar y aùn se ve impedida para sus ocupaciones habituales. He creído mejor hacerlo en una sola y llevarlo hasta su terminación para iniciarlo en la otra. No he intentado la esclerosis para la safena interna por parecerme casi imposible obtener por este método la interrupción completa de la circulación y en algunos casos técnicamente imposible y por no carecer de peligros para la circulación profunda, sin olvidar en los casos favorables la. frecuente recanalización del vaso. Las dificultades son: el espesor.de paniculo adiposo, el contenido considerable de sangre, es una maniobra que se hace al nivel de la desembocadura casi completamente a ciegas y lo puede conducir a la vena femoral e inyectar el líquido en la misma, sin darse cuenta del error, soy partidario de usar el método combinado. El tratamiento combinado tiene por objeto interrumpir definitivamente. el reflujo venoso ligando la vena safena a nivel de su desembocadura en la femoral y las venas colaterales (circunfleja, epigástrica, pudenđa externa). La safena externa debe ligarse en la desembocadura en la poplitea. La ligadura debe ser siempre alta; sin este requisito la recidiva es bastante fácil. Me parece la técnica más segura, ésta: ligadura alta de la safena (no se debe dejar muñon), otra ligadura al nivel del cóndilo femoral interno y ablación del tramo de la safë- 
na comprendido entre las dos incisiones, inyección de sustancia esclerosante en el cabo distal, si no hay esclerosis completa, nueva inyección a los 15 días. La intervención se puede hacer con anestésico local o intravenosa, requiere hospitalización de 5 a 8 días. Se aplica en todo el miembro, por un día, una venda elástica; al tercer día se puede levantar a la paciente, invitándola a dar algunos pasos, el post-operatorio es generalmente apirético.

Los 82 casos tratados con la técnica descrita se pueden agrupar así: en cuanto a la edad: 3 casos entre 18 y 20 años; 20 casos entre 20 y 25 años; 42 casos entre 25 y 30 años; 12 casos entre 30 y 35 años, y 5 casos por encima de esta edad. En cuanto a la prueba de Brodie-Trendelemburg: 39 casos con B. T., igual a 0 (cero); 32 casos con B. T., positivo; 11 casos con B. T., negativo. En cuanto al resultado del tratamiento: 61 casos con resultado muy bueno $(74,39 \%)$; 12 casos con resultado regular $(14,62 \%)$, y 9 casos sin ningún resultado $(10,97 \%)$. Método empleado: 73 esclerosante $(89 \%) ; 9$ combinado $(10 \%)$. Control después del tratamiento: 37 han sido controladas por más de dos años, las 45 restantes no han sido controladas. En cuanto al número de embarazos: 31 , primero; 16, segundo; 20, tercero, y 15, de mayor número.

Estos resultados nos demuestran que si se inicia el tratamiento con B. T. igual a cero o positivo, pueden esperar alto porcentaje en curaciones; basta, por lo tanto, hacer una cuidadosa selección de los casos, como también cuál debe ser el método empleado.

Conclusiones.-19 El tratamiento de las várices venosas no está contraindicado durante los primeros seis meses de embarazo. $2^{\circ}$ El tratamiento esclerosante correctamente establecido es el tratamiento de elección, sin importar la sustancia elegida. $3^{\circ}$ Se debe hacer un estudio clínico completo de cada caso, única manera de poder decidir cuál es el tratamiento apropiado a cada uno. Sin estas bases rigurosamente seguidas se ven más fracasos que éxitos. 4 o $\mathrm{El}$ tratamiento esclerosante no contraindica el posterior quirúrgico, en casos especiales y donde priman antecedentes hereditarios o por razón de la profesión. 50 Establecer como norma este tratamiento precoz de las várices venosas, daría como resultado el cambio de conceptos erróneos establecidos y aparecerían los beneficios que tal conducta traería para las pacientes. 


\section{BIBLIOGRAFIA}

1. EGER S. A.-J. A. M. A

2. BORRIE Y VERNON B.-Brit. Medical J. Número 4.568. Julio 24, 1948.

3. CHISTMANN.-Técnica Quirúrgica. III Tomo. Ateneo. Buenos Aires. 1943.

4. OLIVIER C.-Semain des Hosp. de París. Octubre, 1949.

5. HOMAUS J.-Varix-varicose veins. A test-book of surgery. 1945.

6. TESTUT L.-Tratado de Anatomía Topográfica. IV edición. Tomo II. Salvat, Ed.

7. Villanueva OSORIo A. C.-Prensa Médica Argentina. Número 35. 1948.

8. ORBACH E. J.-J. Intern. Coll. Surg. Número 3. 1950.

9. ROCA J. A.-Faraoni. Día Médico. 1950.

10. SIMPSON H. R.-The inyection treatement of varicose veins. Postgraduate Surg. Volumen II. Appleton Century, N. York. 1938.

11. LAPRENCE A. E.-Várices del miembro inferior. Ed. Ateneo. Buenos Aires. 1951.

12. RECASENS GIROL.-Tratado de Obstetricia. VII Ed. Edit. Nacional México. 1949.

13. SILVA MOJICA CARLOS.-Inestabilidad Tensional y Embarazo. Rev. Col. Obst. Gin. Volumen V. Número 1, 1953.

14. ECHEVERRY G. CORREA H.-Las complicaciones varicosas en la embarazada. Rev. Col. de Obst. Gin. Volumen VI. Número 4. 1955.

15. PERALTA CAYON R.-Obstetricia y Clínica Obstétrica. Tomo I. Antares. 1956.

16. TRIANGULO.-Rev. Sandoz de Cienc. Med. Número 1. Volumen III. 1957.

17. PIULACHS P.-Ulceras de las extremidades inferiores de orden vascular. Salvat. Edit. 1950.

18. BEIGELEINSEN, H. I.-Angiology. Volumen V. Número 2. 1954.

19. PEYRI.-Dermatosis especiales de las extremidades inferiores. Salvat, Edit. 1950.

20. MAIRANO M.-Le vericile 1, ulcera varicosa. Ed. Minerva Médica.

21. MAIRANO M.-Orientamenti attuali sulla terapia delle varici. Ed. Minerva Médica. 1952.

22. MARTORELL.-Várices. Su tratamiento basado en la flebografía. Barcelo. Ed. Labor. 1946. 